\title{
PRESENCIA DE PROSCELIDODON PATRIUS (XENARTHRA, SCELIDOTHERIINAE) EN LA FORMACIÓN CHAPADMALAL (PLIOCENO SUPERIOR), MAR DEL PLATA, BUENOS AIRES, ARGENTINA: IMPLICANCIAS BIOESTRATIGRÁFICAS
}

\author{
MATÍAS TAGLIORETTI ${ }^{1}$, ÁNGEL R. MIÑO-BOILINI ${ }^{2}$, FERNANDO SCAGLIA ${ }^{3}$ Y ALEJANDRO DONDAS ${ }^{3}$
}

${ }^{1}$ Instituto de Geología de Costas, Consejo Nacional de Investigaciones Científicas y Técnicas (CONICET), Deán Funes 3350, B7602AYL Mar del Plata, Argentina. paleomat@yahoo.com.ar

${ }^{2}$ Centro de Ecología Aplicada del Litoral, Consejo Nacional de Investigaciones Científicas y Técnicas (CONICET), Ruta 5, km. 2,5, CC 128, 3400 Corrientes, Argentina. angelmioboilini@yahoo.com.ar

${ }^{3}$ Museo de Ciencias Naturales “Lorenzo Scaglia”, Municipalidad de General Pueyrredón, Av. Libertad 3099, B7600HJB Mar del Plata, Buenos Aires, Argentina. feroscaglia@gmail.com; adondas@gmail.com

Palabras clave. Mammalia. Mylodontidae. Neógeno. Plioceno. Bioestratigrafía.

Key words. Mammalia. Mylodontidae. Neogene. Pliocene. Biostratigraphy.

Los Xenarthra constituyen un grupo particular de mamíferos placentarios e incluyen tres clados: Cingulata, Vermilingua y Tardigrada (= Phyllophaga o Folivora), característicos de la región Neotropical y endémicos del continente americano (Rose y Gaudin, 2010).

Los Tardigrada fósiles están constituidos por cuatros familias: Nothrotheriidae, Megatheriidae, Megalonychidae y Mylodontidae (McDonald y De Iuliis, 2008). Entre los Mylodontidae (Gill, 1872), los Scelidotheriinae (Ameghino, 1904), constituyen el grupo menos diversificado desde el punto de vista taxonómico (McDonald y Perea, 2002). A su vez, los Scelidotheriinae, endémicos de América del Sur, se registran en Argentina desde el Piso/Edad "Friasense" s.l. (Mioceno medio) al Piso/Edad Lujanense (Pleistoceno tardío-Holoceno temprano) (Miño-Boilini, 2012). Según Gaudin (2004), los Scelidotheriinae son un grupo monofilético y, de acuerdo con McDonald y Perea (2002), se caracterizan por presentar un cráneo alargado y estrecho y un astrágalo con una superficie cóncava para la articulación con el cuboides.

El género Proscelidodon Bordas, 1935, se registra para el lapso Huayqueriense-Chapadmalalense Superior de Argentina, Uruguay y Bolivia (Miño-Boilini, 2012). Se reconocen para el género cuatro especies, Proscelidodon patrius (Ameghino, 1888) del Huayqueriense a Chapadmalalense inferior, Proscelidodon almagroi (Rovereto, 1914) y Proscelidodon gra- cillimus (Rovereto, 1914) del Huayqueriense, y Proscelidodon rothi (Kraglievich, 1923) del Chapadmalalense superior.

El objetivo del presente trabajo es dar a conocer y discutir las implicancias bioestratigráficas del primer registro de P. patrius exhumado en sedimentos de la Formación Chapadmalal, en la localidad de Mar del Plata (Buenos Aires, Argentina).

\section{MATERIALES Y MÉTODOS}

El nuevo material estudiado aquí consiste en un cráneo con mandíbula de un individuo juvenil, en buen estado de preservación. Está alojado en el Museo de Ciencias Naturales de Mar del Plata "Lorenzo Scaglia”, con número de colección MMP 4849.

Se realizaron comparaciones con los materiales tipo de $P$. patrius (MACN A-223 y 224, cráneo y mandíbula), P. almagroi (MACN 2544, maxilar parcial derecho), P. gracillimus (MACN 8470, cráneo y mandíbula, deformados por carga litostática) y P. rothi (MLP 3-762, cráneo parcial).

Las observaciones estratigráficas se efectuaron siguiendo a Kraglievich (1952) y Zárate (1989). Para los aspectos bioestratigráficos se siguió el esquema propuesto por Cione y Tonni (2005) y Cione et al. (2007).

Abreviaturas institucionales. MACN, Museo Argentino de Ciencias Naturales "Bernardino Rivadavia", Buenos Aires, 
Argentina; MLP, Museo de La Plata, La Plata, Argentina; MMP, Museo Municipal de Ciencias Naturales "Lorenzo Scaglia", Mar del Plata, Argentina.

Abreviaturas anatómicas. $\mathrm{Mf} / \mathrm{mf}$, molariforme superior $\mathrm{e}$ inferior respectivamente.

Abreviaturas estratigráficas. CHsl, Chapadmalalense sensu lato; $\mathbf{C H i}$, Chapadmalalense inferior; $\mathbf{C H s}$, Chapadmalalense superior; APSC, Aloformación Playa San Carlos; APLL, Aloformación Playa Los Lobos; Fm, Formación.

\section{CONTEXTO GEOGRÁFICO Y ESTRATIGRÁFICO}

La franja costera de los alrededores de la ciudad de Mar del Plata presenta acantilados de 3 a $34 \mathrm{~m}$ de altura, con su sección más potente en el sector sur, constituida por los acantilados conocidos como Barranca de los Lobos, cuyo límite sur está dado por el Arroyo Lobería. Aquí se encuentran los estratotipos de los pisos/edades Chapadmalalense superior (Plioceno superior) y Marplatense (Plioceno superior-Pleistoceno inferior) representados por 4 biozonas (Cione y Tonni, 1995a,b; Cione et al., 2007). Por su parte el Chapadmalalense superior se sustenta en la biozona de $\mathrm{Pa}$ raglyptodon chapadmalalensis (Cione et al., 2007).

Los restos reportados aquí provienen de la localidad Nueva Playa Los Lobos $\left(38^{\circ} 9^{\prime} 8.91^{\prime \prime} \mathrm{S}, 57^{\circ} 37^{\prime} 4.25^{\prime \prime} \mathrm{O}\right.$ ) (Fig. 1), que comprende el sector sur de Barranca de los Lobos. Ameghino (1908) definió el horizonte Chapadmalense en el área costera entre Mar del Plata y Miramar, para incluir una serie de estratos con un contenido faunístico de características intermedias entre las faunas Hermosenses y Ensenadenses. Según Ameghino (1908) el grueso de los afloramientos entre las localidades de Mar del Plata y Miramar está conformado por los horizontes Chapadmalense y Ensenadense, quedando relegados sus horizontes más modernos a los cauces fluviales que disectan la costa. Risso Dominguez (1949a,b) reconoció bajo un criterio estratigráfico nuevos pisos/formaciones entre los horizontes Chapadmalense y Ensenadense, a los cuales denominó desde el más antiguo al más moderno: Chapadketralense, Chapadvorense, Vorohuense, Curalarense, Pequense y Lologhuense. Kraglievich (1952) estableció el concepto actual de "Formación Chapadmalal", utilizando superficies de discontinuidad (ver Reig, 1957) y criterios paleontológicos. Kraglievich (1952) sumó al horizonte Chapadmalense de Ameghino (1908) el piso Chapadketralense de Risso Dominguez (1949a,b).
Posteriormente, Zárate (1989) formalizó, en el marco de la estratigrafía secuencial, el esquema estratigráfico propuesto por Kraglievich (1952), generando un cuadro aloestratigráfico de mayor precisión en el cual los registros paleontológicos pueden ser referidos. Zárate (1989) dividió a la Formación Chapadmalal de Kraglievich (1952) en dos aloformaciones separadas por una discordancia erosiva de extensión local: a) una inferior con cinco paleosuelos, denominada Aloformación Playa San Carlos (APSC) y b) una superior, con dos paleosuelos, denominada Aloformación Playa Los Lobos (APLL). Schultz et al. (1998) realizaron dataciones a partir de escorias provenientes del Paleosuelo 6 de APLL, obteniendo $c a .3,27 \pm 0,08 \mathrm{Ma}$, lo que permitió, a su vez, calibrar la columna magnetoestratigráfica propuesta por Orgeira y Valencio (1984). No existen dataciones absolutas para la APSC, por lo que su edad relativa se deduce de la interpretación magnetoestratigráfica de Orgeira $(1990,1991)$. Zárate (2005) estimó la edad de la "Formación Chapadmalal" (Kraglievich, 1952) entre 4,5 a 3,2 Ma.

La APSC posee un espesor máximo de $12 \mathrm{~m}$ y se caracteriza por un predominio de facies de limolitas con estratificación entrecruzada leve, de geometría tabular y por el desarrollo de paleosuelos representados por horizontes $\mathrm{Bt}$ (Zárate, 1989; Zárate y Fasano, 1989). Las concreciones carbonáticas son escasas y la unidad se encuentra profusamente bioturbada por invertebrados y paleocuevas de vertebrados pequeños, principalmente roedores (Genise, 1989). El espécimen MMP 4849 se recuperó de la porción inferior de la Alocapa 2 de la APSC (Zárate, 1989), comprendida por limolitas tabulares estratificadas (facies lme), caracterizada por la presencia de pequeñas concreciones esféricas de $\mathrm{CO}_{3} \mathrm{Ca}$ con diámetros de entre 10 a $30 \mathrm{~mm}$ y de superficie amamelonada (Fig. 2).

\section{PALEONTOLOGÍA SISTEMÁTICA}

Superorden Xenarthra Cope, 1889

Orden TARDIGRADA (Latham y Davies en Forster, 1795)

Familia Mrlodontidae Gill, 1872

Subfamilia SCELIDOTHERIINAE Ameghino, 1904

Género Proscelidodon Bordas, 1935

Especie tipo. Proscelidodon patrius (Ameghino, 1888a) (ver Mones, 1986).

Proscelidodon patrius (Ameghino, 1888a)

Figura 3, Tabla 1 


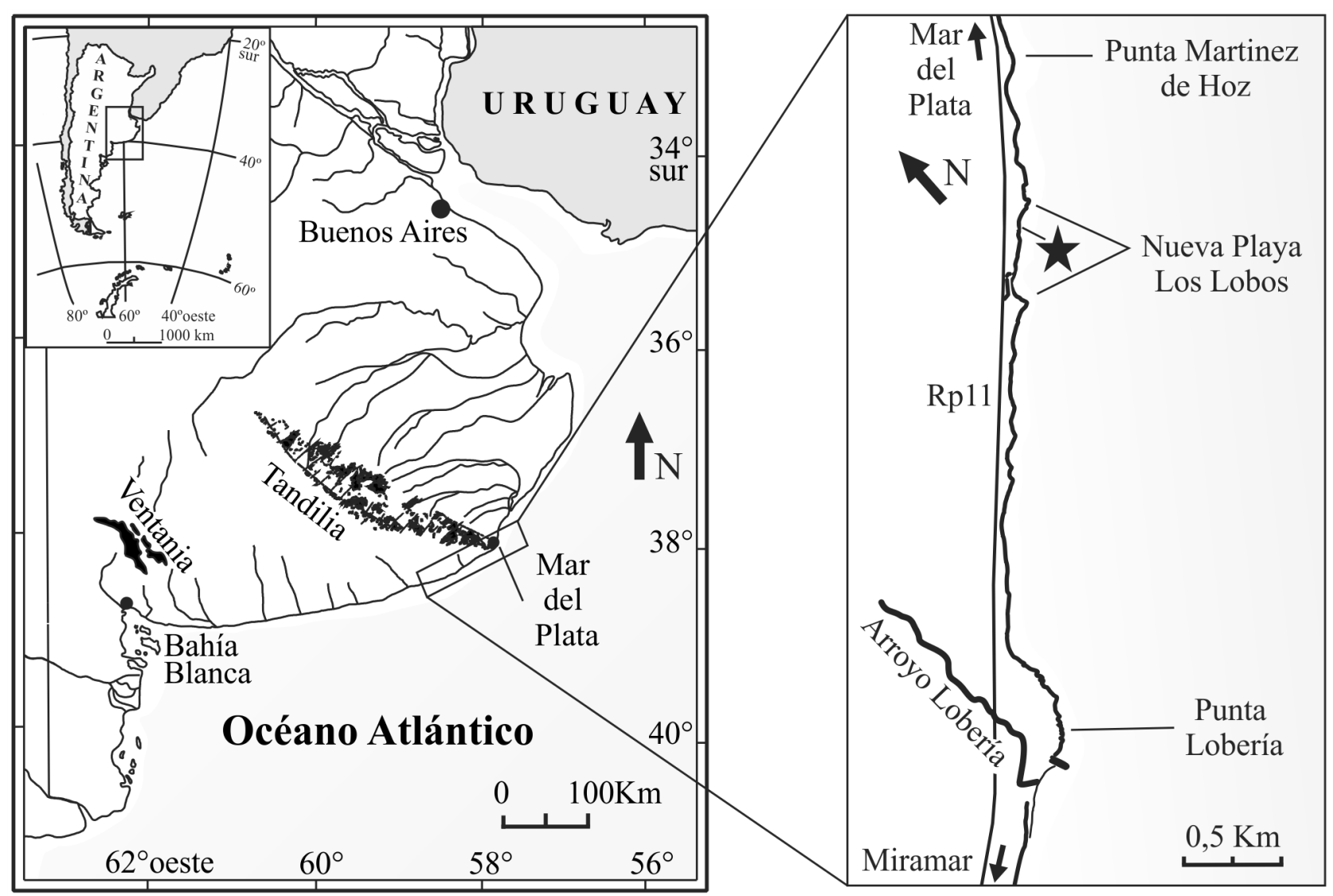

Figura 1. Mapa de la Provincia de Buenos Aires indicando la localidad Nueva Playa Los Lobos donde fue descubierto el espécimen MMP 4849 referido a Proscelidodon patrius.
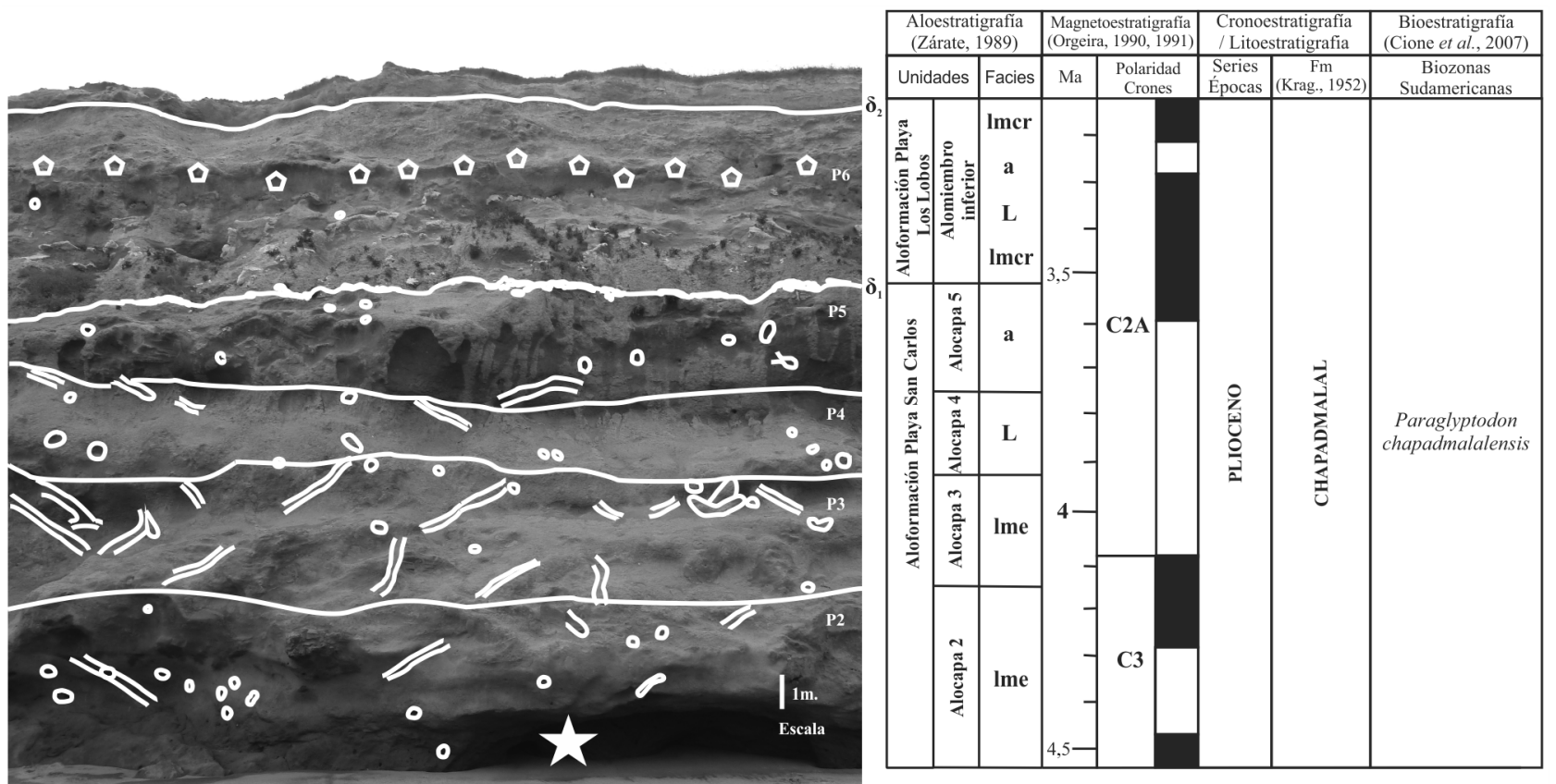

\section{Referencias}

\begin{tabular}{|c|c|c|c|c|c|c|c|c|c|}
\hline $\mathbf{a}$ & Arcillitas & Ime & $\begin{array}{l}\text { Limolitas tabulares } \\
\text { estratificadas }\end{array}$ & $\mathbf{P}$ & Paleosuelos & 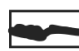 & Banco de tosca macizo & $\delta$ & Discordancia erosiva \\
\hline Imer & $\begin{array}{l}\text { Limolitas arenosas con } \\
\text { estructura de corte y relleno }\end{array}$ & $\mathbf{L}$ & Limos macizos friables & 0 & Paleocuevas & $\theta_{0^{\circ}}$ & $\begin{array}{l}\text { Escorias ca. } 3,27 \pm 0,08 \mathrm{Ma} \\
\text { (Shultz et al., 1998) }\end{array}$ & 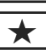 & Proscelidodon patrius \\
\hline
\end{tabular}

Figura 2. Perfil estratigráfico de la Nueva Playa Los Lobos, la estrella indica el nivel donde fue hallado el espécimen MMP 4849. Columna magnetoestratigráfica modificada de Orgeira (1990, 1991). 
Material referido. MMP 4849, cráneo y mandíbula de un individuo juvenil.

Procedencia geográfica y estratigráfica. Nueva Playa Los Lobos, Mar del Plata, Buenos Aires, Argentina $\left(38^{\circ} 9^{\prime} 8.91^{\prime \prime}\right.$, $\left.57^{\circ} 37^{\prime} 4.25^{\prime \prime} \mathrm{O}\right)$. Formación Chapadmalal (Kraglievich, 1952)/ Alocapa 2, Aloformación Playa San Carlos (Zárate, 1989) Plioceno superior.

\section{Descripción y comparación}

Cráneo. El cráneo es estrecho, alargado y posee una longitud aproximada de $185 \mathrm{~mm}$ (desde el extremo posterior de los cóndilos occipitales hasta el extremo anterior del primer diente). Las suturas de los diferentes huesos del cráneo son visibles, y los nasales y maxilares son cortos, como en los géneros Proscelidodon y Catonyx (McDonald, 1987).

En vista anterior (Fig. 3.1), la abertura nasal es de con- torno circular; el extremo anterior de los nasales se proyecta hacia abajo. Esta característica se observa en $P$. patrius y $P$. rothi.

En vista lateral (Fig. 3.2), el cráneo es de contorno subrectangular y presenta los parietales abultados, como es típico en Proscelidodon (McDonald, 1987). La sutura que separa el escamoso, parietal y frontal, es sub-horizontal, característica que se observa en $P$. patrius (en $P$. rothi es horizontal). El proceso cigomático es robusto e inclinado con respecto al eje del Mf3. El lagrimal es oval y presenta un leve contacto con el nasal, similar a P. patrius (McDonald, 1987: figs. 14, 55A,B), diferenciándose de $P$. rothi y $P$. gracillimus en los cuales no se observa un contacto (ver Kraglievich, 1923) debido a que el maxilar en su porción postero-superior posee forma de cuña (McDonald, 1987: fig. 12). El foramen lagrimal es muy notorio como en todos los Scelidotheriinae.
(1)

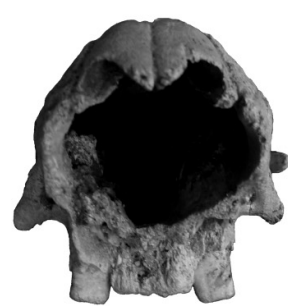

(2)

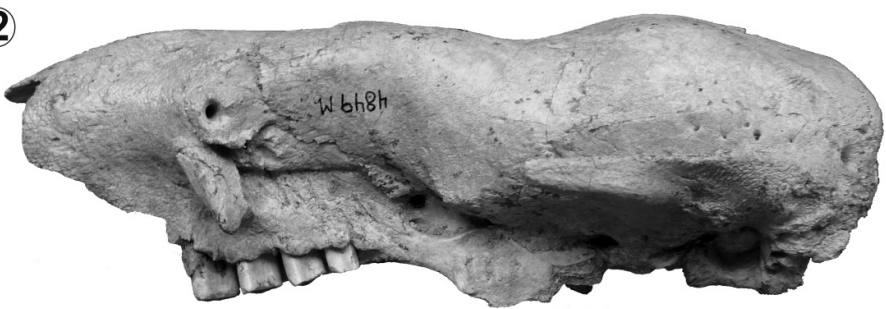

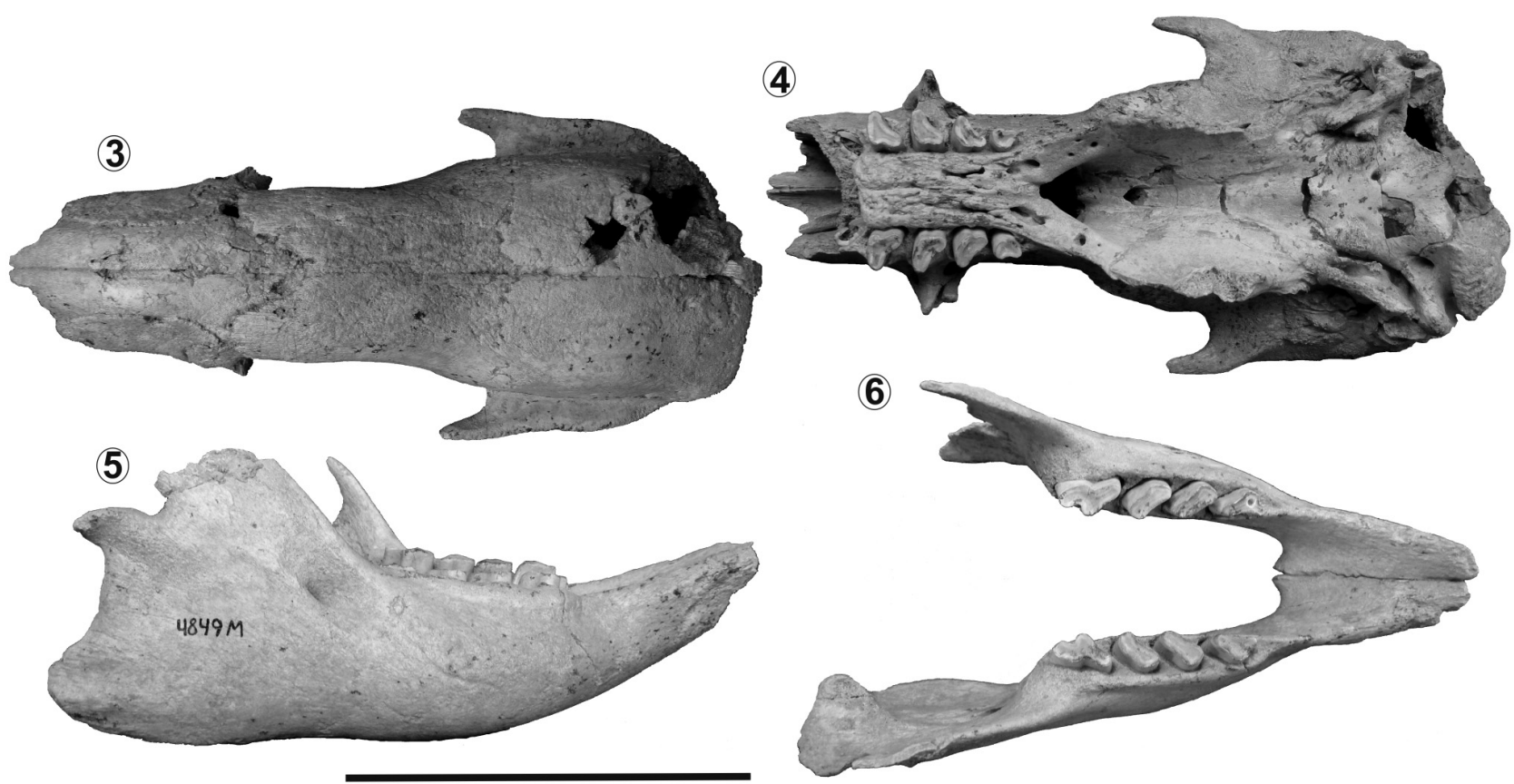

Figura 3. Proscelidodon patrius MMP 4849. 1-4, cráneo; 5-6, mandíbulas. 1, vista anterior; 2, vista lateral izquierda; 3, vista dorsal; 4, vista palatal; 5, vista lateral derecha; 6, vista oclusal. Escala gráfica $=50 \mathrm{~mm}$. 


\begin{tabular}{ll}
\hline \hline $\begin{array}{l}\text { TABLA 1. Dimensiones en mm de los molariformes superiores e inferiores de Proscelidodon patrius (MMP 4849) de la Formación Chapadma- } \\
\text { Ial de Mar del Plata. }\end{array}$ & $9 \times 8$ \\
\hline Diámetro Mf1 (MDXT) & $10 \times 10$ \\
\hline Diámetro Mf2 (MDxT) & $10 \times 11$ \\
\hline Diámetro Mf3 (MDXT) & $9 \times 10$ \\
\hline Diámetro Mf4 (MDXT) & $6 \times 6$ \\
\hline Diámetro Mf5 (MDXT) & 55 \\
\hline Longitud de la serie dentaria superior & $12 \times 7$ \\
\hline Diámetro mf1 (MDxT) & $11 \times 10$ \\
\hline Diámetro mf2 (MDxT) & $11 \times 10$ \\
\hline Diámetro mf3 (MDXT) & $16 \times 9$ \\
\hline Diámetro mf4 (MDxT) & 38 \\
\hline Altura del cuerpo mandibular a nivel del mf3 & 57 \\
\hline Longitud de la serie dentaria inferior &
\end{tabular}

\section{Abreviaturas: $M D$, mesiodistal; $T$, transverso.}

En vista dorsal (Fig. 3.3), el cráneo es de contorno subtriangular como en $P$. patrius (McDonald, 1987: fig. 55 A,B) y $P$. gracillimus, diferenciándose de $P$. rothi que presenta un contorno sub-rectangular (McDonald, 1987, fig: 59). Los nasales y maxilares son cortos, y el proceso anterior de los nasales se extiende más allá del borde anterior de los maxilares, dirigiéndose levemente hacia abajo en su porción más distal.

En vista posterior, el occipital es bajo. El foramen magnum es de contorno ovalado, con su mayor diámetro orientado en sentido transverso.

En vista palatal (Fig. 3.4), las series dentarias son paralelas entre sí como en $P$. gracillimus y $P$. rothi; la sutura entre el maxilar y el palatino se encuentra a nivel del Mf4 como en $P$. patrius, $P$. gracillimus y $P$. rothi (ver además McDonald, 1987: figs. 57A,B, 63 y 59, respectivamente).

El Mf1 es oval, extendido en sentido antero-posterior, como en $P$. patrius. En el caso de $P$. rothi, $P$. almagroi y $P$. gracillimus el Mf1 posee un lóbulo lingual que no se encuentra presente en $P$. patrius (ver Pujos et al., 2012).

El Mf2 muestra un surco longitudinal que define dos lóbulos linguales sub-iguales demarcados; su contorno es subtriangular, similar a $P$. patrius. Sin embargo, en $P$. rothi, $P$. gracillimus y $P$. almagroi es de contorno triangular (ver McDonald, 1987; Pujos et al., 2012).

El Mf3 y el Mf4 también poseen un leve surco longitudinal, menos marcado que en el Mf2, por lo tanto su con- torno es sub-elíptico, similar a $P$. patrius, $P$. gracillimus y $P$. almagroi. En P. rothi el Mf3 y Mf4 son de contorno triangular, con un surco lingual longitudinal muy profundo (Kraglievich, 1923; McDonald, 1987).

El Mf5 presenta un surco longitudinal marcado sobre la cara labial y otro menos marcado en la cara lingual, como en $P$. patrius y $P$. almagroi (ver Ameghino, 1889; Rovereto, 1914). No obstante, en $P$. gracillimus y $P$. rothi es más extendido en sentido antero-posterior (Pujos et al., 2012).

Mandíbula. Faltan el cóndilo articular y los procesos coronoideo y angular izquierdos y el proceso coronoideo derecho. La región predentaria es de longitud sub-igual a la de la serie dentaria (Tab. 1).

En vista lateral (Fig. 3.5), el mayor desarrollo dorso-ventral del cuerpo mandibular se alcanza a nivel del mf3 disminuyendo hacia la porción anterior. El borde ventral es convexo como en todos los Scelidotheriinae del Neógeno (Miño-Boilini et al., 2014). El foramen mental se encuentra en la región anterior de la rama mandibular, como en $P$. $p a-$ trius. El cóndilo mandibular se encuentra levemente por encima del plano oclusal de la serie dentaria. El proceso angular es levemente aguzado, como en $P$. patrius y $P$. gracillimus.

En vista oclusal (Fig. 3.6), la sínfisis mandibular se desarrolla por delante del $\mathrm{mfl}$, como en todos los Scelidotheriinae. La apertura del canal mandibular externo se encuentra por detrás del mf4, como en todos los Scelidotherii- 
nae (McDonald, 1987; Miño-Boilini, 2012).

El mf1 es de sección sub-triangular, con un diámetro labio-lingual menor al mesio-distal (Tab. 1), al igual que en el resto de los Scelidotheriinae (Miño-Boilini, 2012; MiñoBoilini et al., 2014). La cara labial presenta un lóbulo, bien desarrollado y ubicado en la porción media. Esta característica se observa en $P$. patrius y $P$. gracillimus.

El mf2 y mf3 son de contorno sub-cuadrangular, con el diámetro mayor oblicuo al plano sagital (Tab. 1), como en $P$. patrius y $P$. gracillimus.

El mf4 es trilobulado, como en todos los Scelidotheriinae (Miño-Boilini et al., 2014) y más extendido en sentido mesiodistal respecto al resto de los otros dientes. El lóbulo anterior es más extendido en sentido mesio-distal en relación a los otros dos lóbulos, como en P. patrius y P. gracillimus. El lóbulo central se encuentra levemente desarrollado y en posición oblicua con respecto al plano sagital, como es característico de los Scelidotheriinae. Por otro lado, el lóbulo posterior es curvado hacia la cara lingual, como en todos los representantes de la subfamilia (Miño-Boilini et al., 2014).

\section{DISCUSIÓN Y CONCLUSIONES}

El ejemplar MMP 4849 es asignado a $P$. patrius sobre la base de las siguientes características: (1) la sutura entre el escamoso, el parietal y el frontal es sub-horizontal; (2) el lagrimal presenta un leve contacto con el nasal; (3) Mf1 de contorno oval; (4) Mf2 de contorno sub-triangular; y (5) Mf3 y Mf4 de contorno sub-elíptico.

Scillato-Yané et al. (1995) citaron a P. patrius como especie exclusiva de la Edad Montehermosense. Recientemente, Pujos et al. (2012) refirieron nuevos materiales provenientes de la Formación Maimará (Jujuy, Argentina) a P. patrius, extendiendo la base de su biocrón al Huayqueriense (Mioceno tardío). El último registro de $P$. patrius se corresponde a la Biozona de Trigodon gaudryi (Montehermosense; McDonald y Perea, 2002; Miño-Boilini, 2012) en la Formación Montehermoso, Buenos Aires, Argentina (Zavala, 1993), aunque ha sido citado con dudas para la Biozona de Neocavia depressidens (Chapadmalalense inferior; Cione y Tonni, 1995c; Cione y Tonni, 1996), de la misma formación.

Posteriormente Carlini y Scillato-Yané (1999) citaron la presencia de $P$. patrius para la edad Chapadmalalense sensu lato $(\mathrm{CHi}+\mathrm{CH})$, sin referir materiales. Consideramos que este registro es una op cit. de Cione y Tonni (1996). De esta forma Carlini y Scillato-Yané (1999) agregaron un posible registro de P. patrius del CHi (Formación Monte Hermoso, Zavala, 1993) al CHs (= Chapadmalalense sensu stricto= Formación de Chapadmalal, Kraglievich, 1952). Por lo tanto desestimamos este registro previo de $P$. patrius por parte de Carlini y Scillato-Yané (1999) al CHs, por las razones expuestas más arriba. Del mismo modo, Miño-Boilini (2012) citó la presencia de $P$. patrius para el CHsl (op cit. Cione y Tonni, 1996).

En consecuencia, este es el primer registro de $P$. patrius (MMP 4849) para la Formación Chapadmalal (Plioceno superior) de Mar del Plata (Buenos Aires, Argentina) y se suma al de otros Scelidotheriinae, P. rothi (MLP 3-762, tipo) y Scelidotheridium parodii (MACN 5108, tipo), citados para dicha formación (Kraglievich, 1923; McDonald y Perea, 2002). Sin embargo, dada la gran extensión temporal atribuida a dicha formación (4,5-3 Ma, Zárate 2005), la presencia de estas tres especies no puede tomarse por el momento como sincrónica y una revisión detallada de su distribución dentro de los límites de la Formación de Chapadmalal (Kraglievich, 1952) es necesaria.

Para el yacimiento de Farola Monte Hermoso (Buenos Aires), son numerosos los restos recuperados de $P$. patrius (Tonni et al., 1992), en la mayoría de los casos procedentes de los niveles inferiores de la Formación Monte Hermoso (Zavala, 1993), Biozona de Trigodon gaudryi, correspondientes al Montehermosense (Mioceno tardío-Plioceno temprano) (Cione y Tonni, 1995a, 2005; Cione et al., 2007). Recientemente, Tomassini et al. (2013) consideraron como una única unidad estratigráfica a los afloramientos Pliocenos de Farola Monte Hermoso, representada por la biozona de rango de Eumysops laeviplicatus Ameghino, 1888b (Montehermosense). De esta forma se diluye el Chapadmalalense inferior (Cione y Tonni, 1995a) y se restringe la extensión temporal de la edad Montehermosense al intervalo 5,28-5/4,5 Ma.

En este contexto, el hallazgo de P. patrius (MMP 4849) para los niveles más inferiores de la Formación Chapadmalal (APSC, Alocapa 2) invita a replantear la edad de estos depósitos y su posible correlación parcial con los afloramientos de Farola Monte Hermoso (Formación Monte Hermoso). Cabe mencionar que este planteo fue inicialmente propuesto por Pascual et al. (1965) (ver además Prado y Ortiz Jaureguizar, 1989; Tonni et al., 1992; Cione y Tonni, 1995a) quienes no reconocían diferencias significativas entre las faunas Monte- 
hermosenses y Chapadmalalenses, por lo que consideraban sincrónicas las unidades estratigráficas portadores de dichas faunas.

Para poder evaluar la ampliación del biocrón de P. patrius a la edad CHs o eventualmente el cambio de edad de los depósitos portadores del espécimen MMP 4849 al Montehermosense o $\mathrm{CHi}$, es necesario obtener dataciones absolutas o contar con más datos bioestratigráficos que refuercen una u otra hipótesis; de esta forma se evitarán razonamientos circulares en la interpretación de este nuevo dato bioestratigráfico.

\section{AGRADECIMIENTOS}

Agradecemos a A. Kramarz (MACN) y M. Reguero (MLP) por facilitar el acceso a las colecciones bajo su cargo. A V. Taglioretti (FCEYN) y N. Toledo (MLP) por la lectura crítica del manuscrito. Las investigaciones fueron financiadas por el proyecto CONICET PIP 382. A F. Isla (IGCYC) por su constante apoyo. Los autores desean expresar su agradecimiento al editor $\mathrm{S}$. Vizcaíno y a los revisores por sus sugerencias y comentarios sobre el manuscrito que permitieron mejorar esta contribución.

\section{BIBLIOGRAFÍA}

Ameghino, F. 1888a. Rápidas diagnosis de algunos mamiferos fósiles nuevos de la República Argentina. Coni, P.E., Buenos Aires, 17 p.

Ameghino, F. 1888b. Lista de las especies de mamíferos fósiles del Mioceno Superior de Monte Hermoso hasta ahora conocidas. P.E. Coni, Buenos Aires, $21 \mathrm{p}$.

Ameghino, F. 1889. Contribución al conocimiento de los mamíferos fósiles de la República Argentina. Actas de la Academia Nacional de Ciencias de Córdoba 6: 1-1027.

Ameghino, F. 1904. Nuevas especies de mamíferos cretáceos y terciarios de la República Argentina. Anales de la Sociedad Científica Argentina 58: 241-291.

Ameghino, F. 1908. Las formaciones sedimentarias de la región litoral de Mar del Plata y Chapalmalán. Anales del Museo Nacional de Historia Natural de Buenos Aires 3: 343-428.

Bordas, A.F. 1935. Observaciones sobre los géneros "Scelidodon" Ameghino y "Proscelidodon" n. g. Physis 11: 484-491.

Carlini, A.A. y Scillato-Yané, G.J. 1999. Evolution of Quaternary Xenarthrans (Mammalia) of Argentina. En: J. Rabassa and M.C. Salemme (Eds.), Quaternary of South America and Antartic Peninsula. 10: 149176.

Cione, A.L. y Tonni, E.P. 1995a. El estratotipo de los pisos Montehermosense y Chapadmalalense (Plioceno) del esquema cronológico sudamericano. Ameghiniana 32: 369-374.

Cione, A.L. y Tonni, E.P. 1995b. Chronostratigraphy and "Land mammalages": The Uquian problem. Journal of Paleontology 69: 135-159.

Cione, A.L. y Tonni, E.P. 1995c. Bioestratigrafía y cronología del Cenozoico superior de la región pampeana. En: M.T. Alberdi, G. Leone y E.P. Tonni (Eds.), Evolución Biológica y Climática de la Región Pampeana durante los últimos cinco millones de años. Monografías Museo Nacional de Ciencias Naturales, Madrid, p. 49-74.

Cione, A.L. y Tonni, E.P. 1996. Reassessment of the Pliocene-Pleistocene continental time scale of Southern South America. Correlation of the type Chapadmalalan with Bolivian sections. Journal of South American Earth Sciences 9: 221-236.

Cione, A.L. y Tonni, E.P. 2005. Bioestratigrafía basada en mamíferos del Cenozoico superior de la provincia de Buenos Aires, Argentina. $16^{\circ}$ Congreso Geológico Argentino (La Plata), Relatorio: 183-200.

Cione, A.L., Tonni, E.P., Bargo, M.S., Bond, M., Candela, A.M., Carlini, A.A., Deschamps, C.M., Dozo, M.T., Esteban, G., Goin, F.J., Mon- talvo, C.I., Nasif, N., Noriega, J.I., Ortiz Jaureguizar, E., Pascual, R., Prado, J.L., Reguero, M.A., Scillato-Yané, G.J., Soibelzon, L., Verzi, D.H., Vieytes, C., Vizcaíno, F. y Vucetich, M.G. 2007. Mamíferos continentales del Mioceno tardío a la actualidad en la Argentina: cincuenta años de estudios. Ameghiniana, Publicación Especial 11: 257-278.

Cope, E.D. 1889. The Edentata of North America. American Naturalist 23: 657-664.

Gaudin, T.J. 2004. Phylogenetic relationships among sloths (Mammalia, Xenarthra, Tardigrada): the craniodental evidence. Zoological Journal of the Linnean Society 140: 255-305.

Genise, J. 1989. Las cuevas de Actenomys (Rodentia: Octodontidae) de la Formación Chapadmalal (Plioceno Superior) de Mar del Plata y Miramar (Provincia de Buenos Aires). Ameghiniana 26: 33-42.

Gill, T. 1872. Arrangement of the families of mammals with analytical tables. Smithsonian Miscellaneous Collections 11: 1-98.

Latham, J. y Davies, H. 1795. Faunula indica; appendix. En: J.R Forster (Ed.), Zoologia indica, $2^{\text {nd }}$ edition. Editorial Secunda, Halle S. 38 p.

Kraglievich, L. 1923. Descripción comparada de los cráneos de Scelidodon rothi Ameghino y Scelidotherium parodi n. sp. procedentes del horizonte "chapadmalense". Anales del Museo Nacional de Historia Natural "Bernardino Rivadavia" 33: 57-103.

Kraglievich, J.L. 1952. El perfil geológico de Chapadmalal y Miramar, Provincia de Buenos Aires. Revista Museo Municipal Ciencias Naturales y Tradicional Mar del Plata 1: 8-37.

McDonald, H.G. 1987. [A systematic review of the Plio-Pleistocene Scelidotherine Ground Sloth (Mammalia: Xenarthra: Mylodontidae). PhD Tesis, University of Toronto, Toronto, Canada, 478 p. Unpublished.].

McDonald, H.G. y Perea, D. 2002. The large Scelidothere Catonyx tarijensis (Xenarthra, Mylodontidae) from the Pleistocene of Uruguay. Journal of Vertebrate Paleontology 22: 677-683.

McDonald, H.G. y De Iuliis, G. 2008. Fossil history of sloths. En: S.F. Vizcaíno y W.J. Loughry (Eds.), The biology of the Xenarthra. University Press of Florida, Gainesville, p. 39-55.

Miño-Boilini, A.R. 2012. [Sistemática y evolución de los Scelidotheriinae (Xenarthra, Mylodontidae) cuaternarios de la Argentina. Importancia bioestratigráfica, paleobiogeográfica y paleoambiental. Tesis Doctoral, Universidad Nacional de La Plata, La Plata, Argentina, 317 p. Inédita.].

Miño-Boilini, A.R., Tomassini, R.L. y Contreras, V.H. 2014. First record of Scelidotheriinae Ameghino (Xenarthra, Mylodontidae) from the Chasicoan Stage/Age (late Miocene) of Argentina. Estudios Geológicos.

Mones, A. 1986. Paleovertebrata Sudamericana. Catálogo sistemático de los vertebrados fósiles de América del Sur. Parte I. Lista preliminar y Bibliografía. Courier Forschungsinstitut Senckenberg 82: 1-625.

Orgeira, M.J. 1990. Paleomagnetism of late Cenozoic fossiliferous sediments from Barranca de Los Lobos (Buenos Aires Province, Argentina). The Magnetic age of the South American land mammal ages. Physics Earth Planetary Interior 64: 121-132.

Orgeira, M.J. 1991. Correlación magnetoestratigráfica de secuencias cenozoicas tardías de la República Argentina y Bolivia. Asociación Geológica Argentina 46: 127-135.

Orgeira, M.J. y Valencio, D.A. 1984. Estudio paleomagnético de los sedimentos asignados al Cenozoico tardío aflorantes en la Barranca de los Lobos, Provincia de Buenos Aires. $9^{\circ}$ Congreso Geológico Argentino (Bariloche), Actas: 162-173.

Pascual, R., Ortega Hinojosa, E.J., Gondar, D. y Tonni, E.P. 1965. Las edades del Cenozoico mamalífero de la Argentina, con especial atención a aquéllas del territorio bonaerense. Comisión Investigaciones Cientificas de la Provincia de Buenos Aires, Anales 6: 165-193.

Prado, J.L. y Ortiz Jaureguizar, E. 1989. La Edad-mamífero Chapadmalalense: su validez en base a la comparación de su fauna con la de las edades que le precedieron y sucedieron. $\sigma^{\text {as }}$ Jornadas Argentinas Paleontología de Vertebrados (San Juan), Actas: 35-38.

Pujos, F., Candela, A., Galli, C.I., Coira, B.L., Reguero, M.A., de los Reyes, M. y Abello, M.A. 2012. The Scelidotheriine Proscelidodon (Xenarthra: Mylodontidae) from the Late Miocene of Maimará (Northwestern Argentina, Jujuy Province). Ameghiniana 49: 668-674.

Reig, O.A. 1957. Un mustélido del genero Galictis del Eocuartario de la Provincia de Buenos Aires. Ameghiniana 1: 33-47. 
Risso Dominguez, J.D. 1949a. Estratigrafía de las Barrancas de Chapadmalal y Vorohué. Estudios 81: 353-372.

Risso Dominguez, J.D. 1949b. Estratigrafía de las Barrancas de Chapadmalal y Vorohué. Estudios 81: 419-431.

Rose, K.D. y Gaudin, T.J. 2010. Xenarthra and Pholidota (Armadillos, Anteaters, Sloths and Pangolins). En: John Wiley y Sons (Eds.), Encyclopedia of Life Sciences. Ltd. Chichester, p. 1-10.

Rovereto, C. 1914. Los estratos araucanos y sus fósiles. Anales del Museo Nacional de Historia Natural de Buenos Aires 25: 1-247.

Schultz, P.H., Zárate, M., Hames, W., Camilión, C. y King, J. 1998. A 3.3Ma impact in Argentina and possible consequences. Science 282: 20612063.

Scillato-Yane, G.J., Carlini, A.A., Vizcaíno, S.F. y Ortiz Jaureguizar, E. 1995. Los Xenartros. En: M.T. Alberdi, G. Leone y E.P. Tonni (Eds.), Evolución Biológica y Climática de la Región Pampeana durante los últimos cinco millones de años. Monografías Museo Nacional de Ciencias Naturales, Madrid, p. 183-209.

Tomassini, R.L., Montalvo, C. y Deschamps, C. 2013. Biostratigraphy and biochronology of the Monte Hermoso Formation (early Pliocene) at its type locality, Buenos Aires Province, Argentina. Journal of South American Earth Sciences 48: 31-42.

Tonni, E.P., Prado, J., Fidalgo, F. y Laza, J. 1992. El Piso/ Edad Montehermosense (Plioceno) y sus mamíferos. $3^{\text {as }}$ Jornadas Geológicas Bonaerenses (La Plata), Actas: 113-118.

Zárate, M.A. 1989. [Estratigrafía y geología del Cenozoico tardío aflorante en los acantilados marinos comprendidos entre Playa San Carlos y el arroyo Chapadmalal, Partido de General Pueyrredón, Provincia de Buenos Aires. Tesis Doctoral, Facultad de Ciencias Naturales y Museo, Universidad Nacional de La Plata, La Plata, Argentina, 221 p. Inédita.].
Zárate, M.A. 2005. El Cenozoico tardío continental de la Provincia de Buenos Aires. En: R. de Barrio, R. Etcheverry, M. Caballé y E. Llambías (Eds.), Relatorio del $16^{\circ}$ Congreso Geológico Argentino. Geología y Recursos Minerales de la Provincia de Buenos Aires. (La Plata), Relatorio: 139158.

Zárate, M.A. y Fasano, J.L. 1989. The Plio-Pleistocene record of Central Eastern Pampas, Buenos Aires Province, Argentina: The Chapadmalal case study. Palaeogeography, Palaeoclimatology, Palaeoecology 72: 27-52.

Zavala, C. 1993. Estratigrafía de la localidad de Farola Monte Hermoso (Plioceno-Reciente), provincia de Buenos Aires. $12^{\circ}$ Congreso Geológico Argentino y $2^{\circ}$ Congreso de Exploración de Hidrocarburos (Mendoza), Actas: 228-235.

doi: 10.5710/AMGH.04.08.2014.2715

Recibido: 06 de marzo de 2014

Aceptado: 04 de agosto de 2014 